\title{
Erratum to: Deciphering the role of the AT-rich interaction domain and the HMG-box domain of ARID-HMG proteins of Arabidopsis thaliana
}

\author{
Adrita Roy $^{1} \cdot$ Arkajyoti Dutta $^{4} \cdot$ Dipan Roy $^{1} \cdot$ Payel Ganguly $^{1} \cdot$ Ritesh Ghosh $^{2}$. \\ Rajiv K. Kar ${ }^{3} \cdot$ Anirban Bhunia $^{3} \cdot$ Jayanta Mukhopadhyay $^{4} \cdot$ Shubho Chaudhuri $^{1}$
}

Published online: 5 September 2016

(C) Springer Science+Business Media Dordrecht 2016

\section{Erratum to: Plant Mol Biol \\ DOI 10.1007/s11103-016-0519-y}

Due to an unfortunate turn of events, the surname of one of the authors appeared incorrectly in the original publication and should have read Mukhopadhyay.

In addition, an error was introduced in lane 3 of Fig. $6 \mathrm{c}$ showing a negative value of Ligase while it should have indicated a + sign.

The correct representation of the authors' names and the corrected Fig. 6 is published here and should be treated as definitive.

The online version of the original article can be found under doi:10.1007/s11103-016-0519-y.

Shubho Chaudhuri

shubho@jcbose.ac.in; shubho_15@yahoo.com

1 Division of Plant Biology, Bose Institute,

Kolkata 700054, India

2 School of Biotechnology, Yeungnam University, Gyeongsan 712-749, South Korea

3 Department of Biophysics, Bose Institute, Kolkata 700054, India

4 Department of Chemistry, Bose Institute, Kolkata 700054, India 
(A)
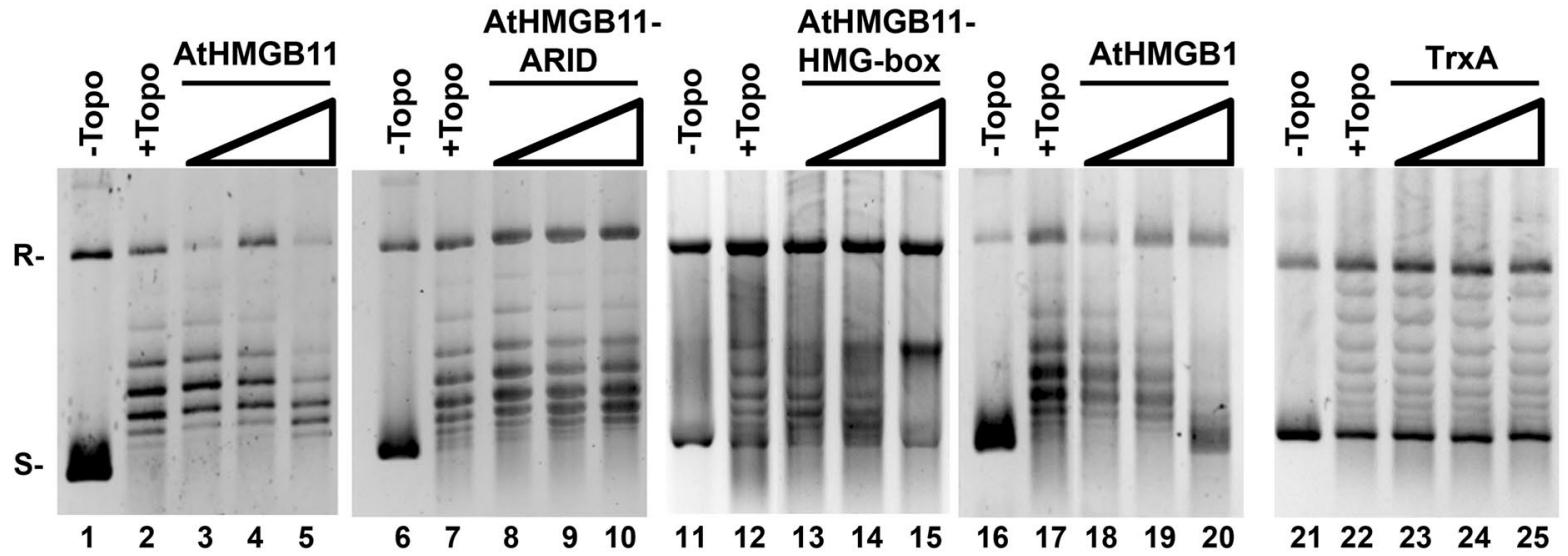

(B)

(C)

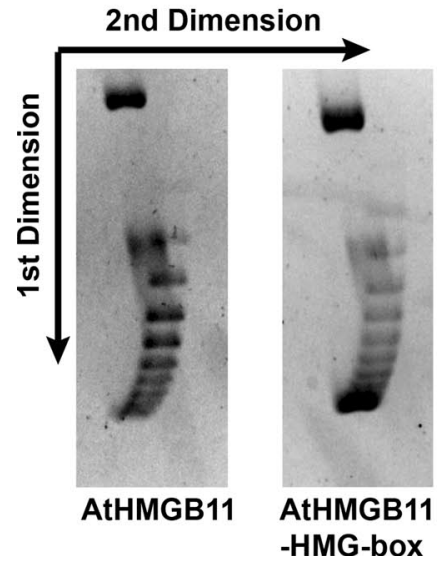

Fig. 6 ARID-HMG can induce change in the DNA topology. a Relaxed pBluescript plasmid was treated with increasing molar input ratio of protein: DNA (10:1, 20:1 and 30:1) each for AtHMGB11, AtHMGB11-ARID, AtHMGB11-HMG-box, AtHMGB1 and TrxA in the presence of topoisomerase I. Lane 2 represents different topoisomeric forms of supercoiled plasmid upon treatment with topoisomerase I. b DNA supercoiling assay done at protein-DNA molar input ratio of 30:1 in the presence of topoisomerase I. A second dimension gel electrophoresis in the presence of chloroquine determines the sign of the superhelical turns introduced by the protein. $\mathbf{c} 120 \mathrm{bp}$ DNA in the presence of T4 DNA ligase shows DNA multimers (lane 2) that are sensitive to exonuclease III digestion (lane 3). The ligation mixture was incubated with protein concentrations $(0.05,0.1,0.2$ and $0.4 \mu \mathrm{M})$ each for AtHMGB11, AtHMGB15, AtHMGB1 and TrxA followed by an exonuclease III digestion for the highest two protein concentrations. Arrowhead indicates exonuclease III resistant circular DNA product in the presence of protein 